\title{
Rates of Antimicrobial Resistance in Latin America (2004-2007) and in vitro Activity of the Glycylcycline Tigecycline and of Other Antibiotics
}

\author{
Flávia Rossi ${ }^{1}$, Patricia García ${ }^{2}$, Bernardo Ronzon $^{3}$, Daniel Curcio ${ }^{4}$, Michael J Dowzicky ${ }^{5}$ \\ ${ }^{1}$ Hospital das Clínicas da Faculdade de Medicina, University of São Paulo, LIM03, Rua Lee de Forest 32, São Paulo, São Paulo, Brazil; \\ ${ }^{2}$ Laboratorio de Microbiología, Departamento de Laboratorios Clínicos, Escuela de Medicina. Pontificia Universidad Católica de Chile; \\ ${ }^{3}$ Hospital Angeles del Pedregal, Camino a Santa Tersa 10 55, col. Heroes de Padierna, Mexico, D.F., Mexico; ${ }^{4}$ Sanatorio San José, \\ Infectología Institucional SRL; Buenos Aires, Argentina; ${ }^{5}$ Wyeth Pharmaceuticals; Collegeville PA, USA
}

\begin{abstract}
As a part of the Tigecycline Evaluation and Surveillance Trial (T.E.S.T.), Gram-positive and Gram-negative bacterial isolates were collected from 33 centers in Latin America (centers in Argentina, Brazil, Chile, Colombia, Guatemala, Honduras, Jamaica, Mexico, Panama, Puerto Rico, and Venezuela) from January 2004 to September 2007. Argentina and Mexico were the greatest contributors of isolates to this study. Susceptibilities were determined according to Clinical Laboratory Standards Institute guidelines. Resistance levels were high for most key organisms across Latin America: $48.3 \%$ of Staphylococcus aureus isolates were methicillin-resistant while $21.4 \%$ of Acinetobacter spp. isolates were imipenem-resistant. Extended-spectrum $\beta$-lactamase were reported in $36.7 \%$ of Klebsiella pneumoniae and $20.8 \%$ of $E$. coli isolates. Tigecycline was the most active agent against Gram-positive isolates. Tigecycline was also highly active against all Gram-negative organisms, with the exception of Pseuodomonas aeruginosa, against which piperacillin-tazobactam was the most active agent tested $(79.3 \%$ of isolates susceptible). The in vitro activity of tigecycline against both Gram-positive and Gram-negative isolates indicates that it may be an useful tool for the treatment of nosocomial infections, even those caused by organisms that are resistant to other antibacterial agents.
\end{abstract}

Key-Words: Antibacterial resistance, Latin America, tigecycline, surveillance.

Increased resistance to antibacterial agents among clinically-important organisms has been widely reported in recent years [1]. In many cases, these organisms are resistant to multiple antibacterial agents [2], dramatically limiting available treatment options. High levels of antibacterial resistance among many key organisms have been reported in Latin America, particularly non-fermentative Gram-negative bacilli (including Acinetobacter spp. and Pseudomonas aeruginosa) and extended-spectrum $\beta$-lactamase (ESBL)producing Enterobacteriaceae, but also among some Grampositive organisms (including Staphylococcus aureus) [3]. Treatment of infections caused by these organisms is often complicated because of resistance to first-line antibacterial agents [4]. Surveillance studies are thus an essential component in the selection of appropriate empirical therapy, allowing for the monitoring of antibacterial resistance [5].

Tigecycline is the first commercially available member of a novel group of antibacterial agents, the glycylcyclines. The Tigecycline Evaluation and Surveillance Trial (T.E.S.T.) is a global surveillance study designed to compare the in vitro activity of tigecycline with a panel of antibacterial agents used in daily clinical practice against a range of clinically important organisms. We report on four-year T.E.S.T. data (2004-2007) for Gram-positive and Gram-negative organisms collected from Latin America.

Received on 6 April 2008; revised 25 September 2008.

Address for correspondence: Dr. Flávia Rossi.Hospital das Clínicas da Faculdade de Medicina. University of São Paulo, LIM03. Rua Lee de Forest 32. São Paulo, São Paulo, Brazil. Phone. 55-11-5505-2480; Fax. 55-11-3758-0457. E-mail: ffrossi@attglobal.net.

The Brazilian Journal of Infectious Diseases 2008;12(5):405-415. (C) 2008 by The Brazilian Journal of Infectious Diseases and Contexto Publishing. All rights reserved.

\section{Material and Methods \\ Isolate Collection}

For the T.E.S.T. study, Latin America is defined as including the following countries: Argentina, Brazil, Chile, Colombia, Guatemala, Honduras, Jamaica, Mexico, Panama, Puerto Rico and Venezuela (Table 1). Thirty-three Latin American centers participated in the T.E.S.T. study from January 2004 to September 2007 (Table 1). Data for 2007 are as yet incomplete.

Isolates that were determined by local criteria to be clinically significant were collected from in- and out-patients with documented nosocomial or community-acquired infections. Acceptable isolate sources included blood, the respiratory tract, urine (not exceeding $25 \%$ of isolates), skin, wounds and fluids. Only one isolate per patient was accepted in the study. Each center was required to contribute the following: 25 isolates each of Staphylococcus aureus, Klebsiella spp., Escherichia coli and Enterobacter spp.; 20 isolates of Pseudomonas aeruginosa; 15 isolates each of Enterococcus spp. and Acinetobacter spp.; and 10 isolates of Serratia marcescens. A maximum of 200 isolates was contributed by each center, after local determination of identity and antibacterial susceptibility.

\section{Antimicrobial Susceptibility Testing}

Minimum inhibitory concentrations (MICs) were determined locally using broth microdilution methodology, as defined by the Clinical Laboratory Standards Institute (CLSI, formerly NCCLS) [6], using either MicroScan ${ }^{\circledR}$ panels (Dade Behring Inc., CA, USA) or Sensititre ${ }^{\circledR}$ plates (TREK Diagnostic Systems, West Sussex, England). The test panel for Gramnegative isolates included (concentrations given in $\mu \mathrm{g} / \mathrm{mL}$ ): amikacin (0.5-64); amoxicillin-clavulanic acid (0.12/0.06-32/16); 
ampicillin (0.5-32); cefepime (0.5-32); ceftriaxone (0.06-64); ceftazidime (8-32); imipenem (0.06-16, MicroScan ${ }^{\circledR}$ only); levofloxacin (0.008-8); minocycline (0.5-16); tigecycline (0.00816); and piperacillin-tazobactam (0.06/4-128/4). Gram-positive organisms were tested against the following agents (in $\mu \mathrm{g} / \mathrm{mL}$ ): amoxicillin-clavulanic acid (0.03/0.0015-8/4), ampicillin (0.06-16), penicillin(0.06-8), linezolid(0.5-8), ceftriaxone(0.03-64), imipenem (0.12-16, MicroScan ${ }^{\circledR}$ only), levofloxacin (0.06-32), minocycline (0.25-8), tigecycline (0.008-16), piperacillin-tazobactam (0.25/416/4), and vancomycin (0.12-32). For tigecycline, interpretive criteria from the US Food and Drug Administration packaging insert were applied for $S$. aureus (susceptible $\leq 0.5 \mu \mathrm{g} / \mathrm{mL}$ ), vancomycin-susceptible $E$. faecalis (susceptible $\leq 0.25 \mu \mathrm{g} / \mathrm{mL}$ ) and the Enterobacteriaceae (susceptible $\leq 2 \mu \mathrm{g} / \mathrm{mL}$, intermediate $4 \mu \mathrm{g} / \mathrm{mL}$, resistant $\geq 8 \mu \mathrm{g} / \mathrm{mL}$ ) [7]. For all other agents, CLSI interpretive criteria were applied [8].

MIC determinations were carried out using cation-adjusted Mueller-Hinton broth. Broth microdilution panels inoculated with Gram-negative organisms were incubated in ambient air at $35^{\circ} \mathrm{C}$ for $16-20$ hours. Staphylococcus aureus and Enterococcus spp. were incubated in ambient air at $35^{\circ} \mathrm{C}$ for 20-24 hours. Quality control testing was carried out on each day of susceptibility testing, using the following ATCC strains: E. coli ATCC 25922 and 35218, S. aureus ATCC 29213, P. aeruginosa ATCC 27853, and E. faecalis ATCC 29212, when appropriate.

All isolates were sent to a single laboratory, International Health Management Associates, Inc. (IHMA, Schaumburg, IL, US), where confirmation of identification was carried out as well as inclusion of all data into a centralized database. MIC information was also returned to the central laboratory for inclusion in this centralized database using an OptiScan Data Collection Form, part of a proprietary optical character recognition system. MICs of approximately $10 \%$ of isolates from all centers were checked by IHMA as a part of standard quality control testing.

Antibacterial Resistance Determination

Confirmation of methicillin resistance was carried out by IHMA using the cefoxitin disk diffusion method, using $30 \mu \mathrm{g}$ disks obtained from Remel, Lenexa, KS, USA.

The presence of ESBLs among K. pneumoniae and E. coli was detected according to CLSI methodology [8], for which cefotaxime (30 $\mu \mathrm{g})$, cefotaxime-clavulanic acid (30/10 $\mu \mathrm{g})$, ceftazidime (30 $\mu \mathrm{g})$ and ceftazidime-clavulanic acid (30/10 $\mu \mathrm{g})$ discs were used. An increase of $\geq 5 \mathrm{~mm}$ in inhibition zone on the combination disc compared to the cephalosporin only disc suggested an ESBL producing organism.

\section{Results}

The incidence of organisms, both resistant and susceptible, varied widely between species and between countries during the four-year study period. Most isolates came from Argentina or Mexico during this study interval (Tables 2 and 3). 


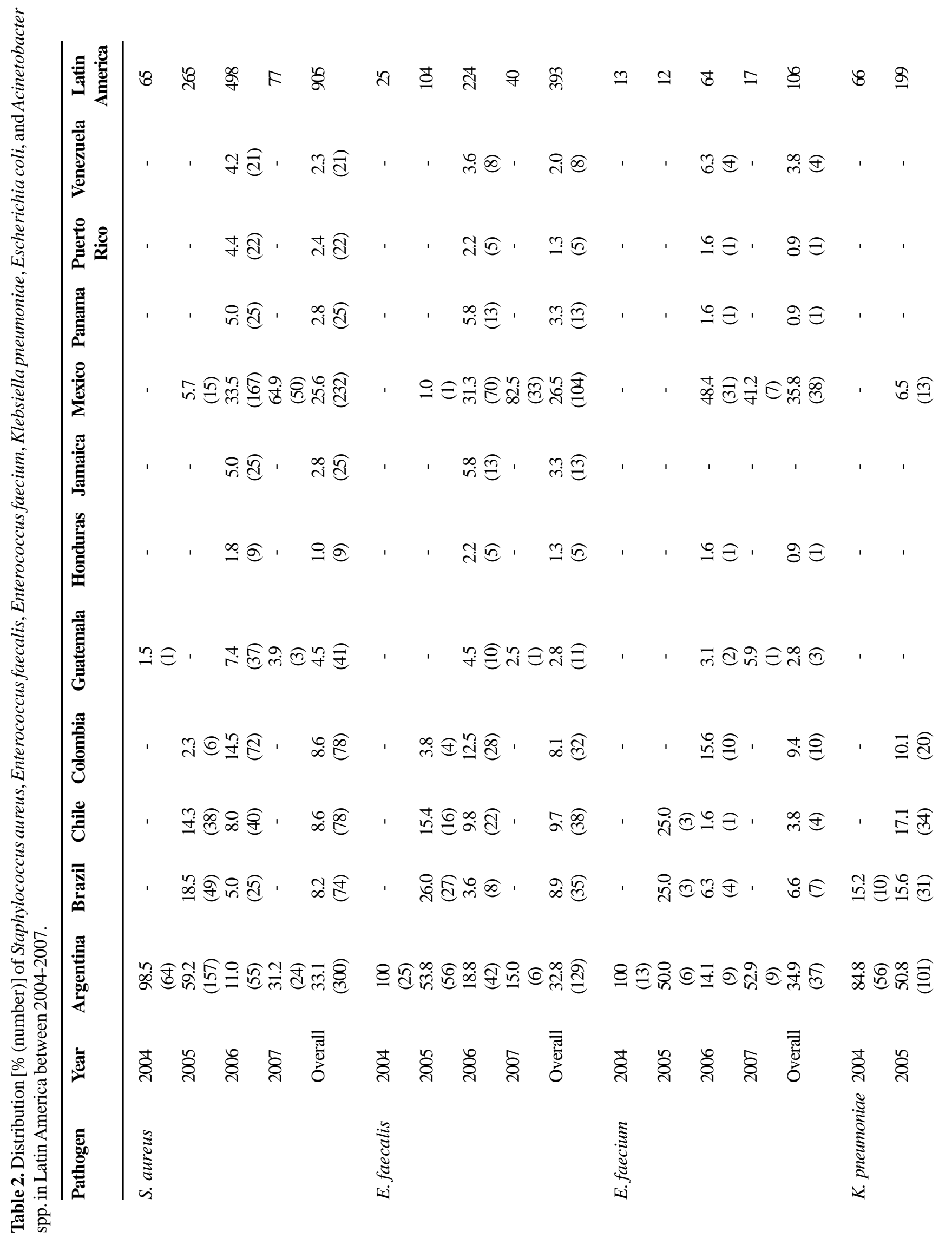




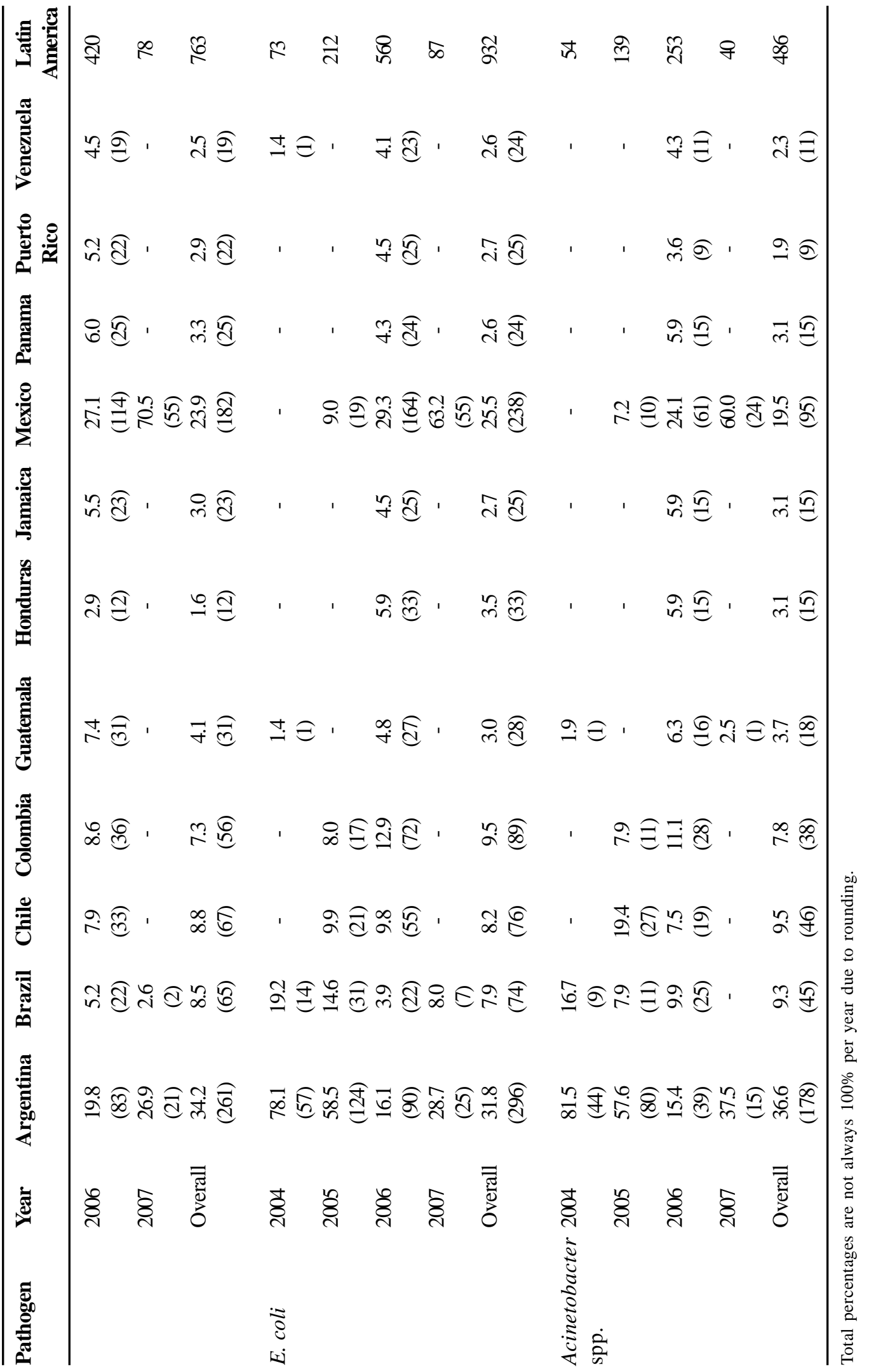


Gram-Positive Isolates

Staphylococcus aureus

All 905 S. aureus isolates collected between 2004 and 2007 were susceptible to tigecycline, linezolid and vancomycin $\left(\mathrm{MICs}_{90} 0.25,2\right.$ and $1 \mu \mathrm{g} / \mathrm{mL}$, respectively), while $98.9 \%$ were susceptible to minocycline ( $\mathrm{MIC}_{90} 1 \mu \mathrm{g} / \mathrm{mL}$ ) (Table 4). Only $55.7 \%$ of isolates were susceptible to levofloxacin. Almost one-half of these S. aureus isolates (48.3\%) were methicillinresistant (MRSA) (Table 3).

\section{Enterobacter faecalis and E. faecium}

All E. faecalis isolates were susceptible to ampicillin, while most isolates were susceptible to penicillin (99.2\%), linezolid (97.2\%) and vancomycin (96.4\%). Low $\mathrm{MICs}_{90}$ were reported for tigecycline and amoxicillin-clavulanic acid (0.25 and $1 \mu \mathrm{g} /$ $\mathrm{mL}$, respectively; Table 4).

Against E. faecium the lowest MICs were reported for tigecycline $(0.12 \mu \mathrm{g} / \mathrm{mL})$, while $92.5 \%$ of isolates were susceptible to linezolid ( $\mathrm{MIC}_{90} 2 \mu \mathrm{g} / \mathrm{mL}$ ). Only $17.0 \%$ of isolates were susceptible to levofloxacin ( $\mathrm{MIC}_{90} \geq 64 \mu \mathrm{g} / \mathrm{mL}$, Table 4). Nearly half (44.3\%) of the E. faecium isolates observed in this study were vancomycin resistant (Table 3).

\section{Gram-Negative Isolates Non-Fermenters}

Against the Acinetobacter spp. the lowest $\mathrm{MICs}_{90}$ were reported for tigecycline and minocycline $(2 \mu \mathrm{g} / \mathrm{mL})$, with $94.9 \%$ of isolates susceptible to minocycline. Only $62.6 \%$ of isolates were susceptible to imipenem ( $\mathrm{MIC}_{90} \geq 32 \mu \mathrm{g} / \mathrm{mL}$, Table 4).

Few agents were active against $P$. aeruginosa. Piperacillintazobactam was the most active agent reported against $P$. aeruginosa in this study, with $79.3 \%$ of isolates susceptible over the four study years $\left(\mathrm{MIC}_{90} 128 \mu \mathrm{g} / \mathrm{mL}\right.$, Table 4 ).

\section{Enterobacteriaceae}

Imipenem and tigecycline were the most active agents against Enterobacter spp., with 99.2 and $96.2 \%$ of isolates susceptible, respectively. Susceptibility to cephalosporins ranged from $58.0 \%$ to ceftazidime to $80.9 \%$ to cefepime (Table 4).

High susceptibilities were reported among isolates of $K$. pneumoniae against imipenem (99.6\%; $\left.\mathrm{MIC}_{90} 1 \mu \mathrm{g} / \mathrm{mL}\right)$ and tigecycline (95.7\%; $\mathrm{MIC}_{90} 2 \mu \mathrm{g} / \mathrm{mL}$ ) (Table 4).

Tigecycline was the most active agent against $E$. coli; all isolates were susceptible $\left(\mathrm{MIC}_{90} 0.5 \mu \mathrm{g} / \mathrm{mL}\right.$ ). Other active agents included imipenem (99.7\% susceptible), amikacin (97.3\% susceptible) and piperacillin-tazobactam (91.0\% susceptible) (Table 4).

Among isolates of S. marcescens, 99.5\% were susceptible to imipenem, while $97.3 \%$ were susceptible to tigecycline (Table 4 ).

\section{Resistant Isolates}

Tigecycline, vancomycin, linezolid and minocycline were effective against MRSA, with $\mathrm{MICs}_{90}$ of $0.25,1,2$ and $4 \mu \mathrm{g} /$ $\mathrm{mL}$, respectively. Susceptibilities to these agents were also 
Table 4. In vitro activity of tigecycline and other antibiotics against Gram-positive and Gram-negative organisms collected in Latin America from 2004-2007.

\begin{tabular}{|c|c|c|c|c|c|c|}
\hline \multirow[b]{2}{*}{ Antibacterial } & \multicolumn{6}{|c|}{ Overall (2004-2007) } \\
\hline & $\mathbf{N}$ & $\mathrm{MIC}_{50}$ & $\mathrm{MIC}_{90}$ & $\% \mathrm{~S} *$ & $\% \mathbf{R}^{*}$ & MIC range \\
\hline \multicolumn{7}{|l|}{ Gram positives } \\
\hline \multicolumn{7}{|l|}{ S. aureus } \\
\hline Tigecycline & 905 & 0.12 & 0.25 & 100 & N/A & $0.03-0.5$ \\
\hline Penicillin & 905 & $\geq 16$ & $\geq 16$ & 6.5 & 93.5 & $\leq 0.06-\geq 16$ \\
\hline Ampicillin & 905 & 16 & $\geq 32$ & 8.4 & 91.6 & $\leq 0.06-\geq 32$ \\
\hline Amoxicillin-clavulanic acid & 905 & 2 & $\geq 16$ & 57.7 & 42.3 & $0.06-\geq 16$ \\
\hline Piperacillin-tazobactam & 905 & 2 & $\geq 32$ & 59.4 & 40.6 & $\leq 0.25-\geq 32$ \\
\hline Ceftriaxone & 905 & 4 & $\geq 128$ & 54.7 & 39.4 & $0.12-\geq 128$ \\
\hline Imipenem $^{\dagger}$ & 538 & 0.25 & $\geq 32$ & 59.5 & 36.8 & $\leq 0.12-\geq 32$ \\
\hline Levofloxacin & 905 & 0.25 & 16 & 55.7 & 41.2 & $\leq 0.06-\geq 64$ \\
\hline Minocycline & 905 & $\leq 0.25$ & 1 & 98.9 & 0.2 & $\leq 0.25-\geq 16$ \\
\hline Linezolid & 905 & 2 & 2 & 100 & N/A & $\leq 0.5-4$ \\
\hline Vancomycin & 905 & 1 & 1 & 100 & 0.0 & $\leq 0.12-2$ \\
\hline \multicolumn{7}{|l|}{ E. faecalis } \\
\hline Tigecycline & 393 & 0.12 & 0.25 & N/A & N/A & $\leq 0.008-1$ \\
\hline Penicillin & 393 & 2 & 4 & 99.2 & 0.8 & $\leq 0.06-\geq 16$ \\
\hline Ampicillin & 393 & 1 & 2 & 100 & 0.0 & $\leq 0.06-8$ \\
\hline Amoxicillin-clavulanic acid & 393 & 0.5 & 1 & N/A & N/A & $0.06-8$ \\
\hline Piperacillin-tazobactam & 393 & 2 & 8 & N/A & N/A & $\leq 0.25-\geq 32$ \\
\hline Imipenem $^{\dagger}$ & 229 & 1 & 8 & N/A & N/A & $\leq 0.12-\geq 32$ \\
\hline Levofloxacin & 393 & 1 & 32 & 71.0 & 27.7 & $0.25-\geq 64$ \\
\hline Minocycline & 393 & 8 & $\geq 16$ & 38.7 & 24.4 & $\leq 0.25-\geq 16$ \\
\hline Linezolid & 393 & 2 & 2 & 97.2 & 0.0 & $\leq 0.5-4$ \\
\hline Vancomycin & 393 & 1 & 2 & 96.4 & 2.8 & $\leq 0.12-\geq 64$ \\
\hline \multicolumn{7}{|l|}{ E. faecium } \\
\hline Tigecycline & 106 & 0.06 & 0.12 & 100 & 0.0 & $\leq 0.008-0.25$ \\
\hline Penicillin & 106 & $\geq 16$ & $\geq 16$ & 22.6 & 77.4 & $\leq 0.06-\geq 16$ \\
\hline Ampicillin & 106 & $\geq 32$ & $\geq 32$ & 25.5 & 74.5 & $0.12-\geq 32$ \\
\hline Amoxicillin-clavulanic acid & 106 & $\geq 16$ & $\geq 16$ & N/A & N/A & $0.12-\geq 16$ \\
\hline Piperacillin-tazobactam & 106 & $\geq 32$ & $\geq 32$ & N/A & N/A & $\leq 0.25-\geq 32$ \\
\hline Ceftriaxone & 106 & $\geq 128$ & $\geq 128$ & N/A & N/A & $0.12-\geq 128$ \\
\hline Imipenem $^{\dagger}$ & 54 & $\geq 32$ & $\geq 32$ & N/A & N/A & $0.5-\geq 32$ \\
\hline Levofloxacin & 106 & $\geq 64$ & $\geq 64$ & 17.0 & 78.3 & $0.5-\geq 64$ \\
\hline Minocycline & 106 & 1 & $\geq 16$ & 61.3 & 22.6 & $\leq 0.25-\geq 16$ \\
\hline Linezolid & 106 & 2 & 2 & 92.5 & 0.0 & $\leq 0.5-4$ \\
\hline Vancomycin & 106 & 2 & $\geq 64$ & 53.8 & 44.3 & $\leq 0.12-\geq 64$ \\
\hline \multicolumn{7}{|l|}{ Gram negatives } \\
\hline \multicolumn{7}{|l|}{ Acinetobacter spp. } \\
\hline Tigecycline & 486 & 0.5 & 2 & N/A & N/A & $\leq 0.008-8$ \\
\hline Tigecycline & 486 & 0.5 & 2 & 96.7 & 0.4 & $\leq 0.008-8$ \\
\hline \multicolumn{7}{|c|}{ (Enterobacteriaceae breakpoints ${ }^{¥}$ ) } \\
\hline Ampicillin & 486 & $\geq 64$ & $\geq 64$ & N/A & N/A & $\leq 0.5-\geq 64$ \\
\hline Amoxicillin-clavulanic acid & 486 & $\geq 64$ & $\geq 64$ & N/A & N/A & $\leq 0.12-\geq 64$ \\
\hline Piperacillin-tazobactam & 486 & 128 & $\geq 256$ & 19.1 & 62.3 & $\leq 0.06-\geq 256$ \\
\hline Ceftazidime & 486 & $\geq 64$ & $\geq 64$ & 18.1 & 75.1 & $\leq 8-\geq 64$ \\
\hline
\end{tabular}




\begin{tabular}{|c|c|c|c|c|c|c|}
\hline Ceftriaxone & 486 & $\geq 128$ & $\geq 128$ & 8.4 & 79.4 & $\leq 0.06-\geq 128$ \\
\hline Cefepime & 486 & 32 & $\geq 64$ & 25.9 & 54.1 & $\leq 0.5-\geq 64$ \\
\hline Imipenem $^{\dagger}$ & 313 & 1 & $\geq 32$ & 62.6 & 33.2 & $0.12-\geq 32$ \\
\hline Levofloxacin & 486 & 8 & $\geq 16$ & 19.5 & 62.1 & $\leq 0.008-\geq 16$ \\
\hline Amikacin & 486 & 32 & $\geq 128$ & 35.6 & 46.1 & $\leq 0.5-\geq 128$ \\
\hline Minocycline & 486 & $\leq 0.5$ & 2 & 94.9 & 2.1 & $\leq 0.5-\geq 32$ \\
\hline \multicolumn{7}{|l|}{ P. aeruginosa } \\
\hline Tigecycline & 715 & 8 & $\geq 32$ & N/A & N/A & $0.12-\geq 32$ \\
\hline Ampicillin & 715 & $\geq 64$ & $\geq 64$ & N/A & N/A & $1-\geq 64$ \\
\hline Piperacillin-tazobactam & 715 & 8 & 128 & 79.3 & 20.7 & $\leq 0.06-\geq 256$ \\
\hline Ceftazidime & 715 & $\leq 8$ & $\geq 64$ & 60.0 & 30.5 & $\leq 8-\geq 64$ \\
\hline Cefepime & 715 & 8 & $\geq 64$ & 61.5 & 22.8 & $\leq 0.5-\geq 64$ \\
\hline Imipenem $^{\dagger}$ & 446 & 1 & 16 & 66.1 & 18.8 & $0.12-\geq 32$ \\
\hline Levofloxacin & 715 & 2 & $\geq 16$ & 50.5 & 45.0 & $0.03-\geq 16$ \\
\hline Amikacin & 715 & 4 & 64 & 72.2 & 18.5 & $\leq 0.5-\geq 128$ \\
\hline Minocycline & 715 & $\geq 32$ & $\geq 32$ & 5.2 & 79.6 & $\leq 0.5-\geq 32$ \\
\hline \multicolumn{7}{|l|}{ Enterobacter spp. } \\
\hline Tigecycline & 766 & 0.5 & 2 & 96.2 & 0.3 & $0.06-8$ \\
\hline Ampicillin & 766 & $\geq 64$ & $\geq 64$ & 0.4 & 93.5 & $4-\geq 64$ \\
\hline Amoxicillin-clavulanic acid & 766 & $\geq 64$ & $\geq 64$ & 3.3 & 94.6 & $1-\geq 64$ \\
\hline Piperacillin-tazobactam & 766 & 4 & 128 & 68.4 & 15.5 & $0.12-\geq 256$ \\
\hline Ceftazidime & 766 & $\leq 8$ & $\geq 64$ & 58.0 & 36.2 & $\leq 8-\geq 64$ \\
\hline Ceftriaxone & 766 & 1 & $\geq 128$ & 61.6 & 28.5 & $\leq 0.06-\geq 128$ \\
\hline Cefepime & 766 & $\leq 0.5$ & $\geq 64$ & 80.9 & 14.5 & $\leq 0.5-\geq 64$ \\
\hline Imipenem $^{\dagger}$ & 484 & 0.5 & 1 & 99.2 & 0.6 & $\leq 0.06-\geq 32$ \\
\hline Levofloxacin & 766 & 0.06 & $\geq 16$ & 76.6 & 19.6 & $\leq 0.008-\geq 16$ \\
\hline Amikacin & 766 & 2 & 32 & 89.4 & 5.4 & $\leq 0.5-\geq 128$ \\
\hline Minocycline & 766 & 4 & $\geq 32$ & 72.5 & 17.6 & $\leq 0.5-\geq 32$ \\
\hline \multicolumn{7}{|l|}{ K. pneumoniae } \\
\hline Tigecycline & 763 & 0.5 & 2 & 95.7 & 1.3 & $0.12-8$ \\
\hline Ampicillin & 763 & $\geq 64$ & $\geq 64$ & 0.3 & 90.4 & $1-\geq 64$ \\
\hline Amoxicillin-clavulanic acid & 763 & 16 & $\geq 64$ & 49.1 & 35.0 & $0.5-\geq 64$ \\
\hline Piperacillin-tazobactam & 763 & 4 & $\geq 256$ & 66.1 & 22.9 & $0.12-\geq 256$ \\
\hline Ceftazidime & 763 & $\leq 8$ & $\geq 64$ & 56.7 & 35.3 & $\leq 8-\geq 64$ \\
\hline Ceftriaxone & 763 & 1 & $\geq 128$ & 56.7 & 35.6 & $\leq 0.06-\geq 128$ \\
\hline Cefepime & 763 & 1 & $\geq 64$ & 67.6 & 26.5 & $\leq 0.5-\geq 64$ \\
\hline Imipenem $^{\dagger}$ & 459 & 0.5 & 1 & 99.6 & 0.4 & $\leq 0.06-\geq 32$ \\
\hline Levofloxacin & 763 & 0.25 & $\geq 16$ & 63.4 & 32.2 & $\leq 0.008-\geq 16$ \\
\hline Amikacin & 763 & 2 & 32 & 88.3 & 7.5 & $\leq 0.5-\geq 128$ \\
\hline Minocycline & 763 & 2 & 16 & 72.6 & 18.1 & $\leq 0.5-\geq 32$ \\
\hline \multicolumn{7}{|l|}{ E. coli } \\
\hline Tigecycline & 932 & 0.25 & 0.5 & 100 & 0.0 & $\leq 0.008-2$ \\
\hline Ampicillin & 932 & $\geq 64$ & $\geq 64$ & 25.6 & 73.3 & $\leq 0.5-\geq 64$ \\
\hline Amoxicillin-clavulanic acid & 932 & 8 & 32 & 56.0 & 20.0 & $\leq 0.12-\geq 64$ \\
\hline Piperacillin-tazobactam & 932 & 1 & 16 & 91.0 & 4.1 & $\leq 0.06-\geq 256$ \\
\hline Ceftazidime & 932 & $\leq 8$ & 32 & 77.9 & 12.4 & $\leq 8-\geq 64$ \\
\hline Ceftriaxone & 932 & $\leq 0.06$ & $\geq 128$ & 71.0 & 23.9 & $\leq 0.06-\geq 128$ \\
\hline Cefepime & 932 & $\leq 0.5$ & 32 & 80.4 & 14.6 & $\leq 0.5-\geq 64$ \\
\hline Imipenem $^{\dagger}$ & 583 & 0.25 & 0.5 & 99.7 & 0.2 & $\leq 0.06-\geq 32$ \\
\hline Levofloxacin & 932 & 0.5 & $\geq 16$ & 53.8 & 41.2 & $\leq 0.008-\geq 16$ \\
\hline Amikacin & 932 & 2 & 8 & 97.3 & 1.3 & $\leq 0.5-\geq 128$ \\
\hline Minocycline & 932 & 2 & 16 & 68.3 & 18.7 & $\leq 0.5-\geq 32$ \\
\hline
\end{tabular}




\begin{tabular}{|c|c|c|c|c|c|c|}
\hline \multicolumn{7}{|l|}{ S. marcescens } \\
\hline \multicolumn{7}{|l|}{$\begin{array}{l}\text { Tigecycline } \\
\text { Tigarcens }\end{array}$} \\
\hline \multicolumn{7}{|l|}{ Ampicillin } \\
\hline \multicolumn{7}{|l|}{$\begin{array}{l}\text { Tigecycline } \\
\text { Ampicillin } \\
\text { Amoxicillin-clavulanic acid }\end{array}$} \\
\hline Piperacillin-tazobactam & 328 & 2 & 128 & 80.2 & 11.0 & $\leq 0.06-\geq 256$ \\
\hline \multicolumn{7}{|l|}{ Ceftazidime } \\
\hline \multicolumn{7}{|l|}{ Ceftriaxone } \\
\hline \multicolumn{7}{|r|}{$\leq 0.5-\geq 64$} \\
\hline \multicolumn{7}{|l|}{ Imipenem $^{\dagger}$} \\
\hline Levofloxacin & 328 & 0.12 & 4 & 84.5 & 9.8 & $\leq 0.008-\geq 16$ \\
\hline Amikacin & 328 & 4 & 32 & 79.9 & 9.8 & $\leq 0.5-\geq 128$ \\
\hline Minocycline & 328 & 2 & 8 & 85.7 & 5.8 & $\leq 0.5-\geq 32$ \\
\hline
\end{tabular}

*Susceptibility and resistance were only determined when $\geq 10$ isolates were available; ${ }^{\dagger}$ Imipenem numbers are lower due to some isolates being tested with meropenem (data not shown); ${ }^{*}$ using Enterobacteriaceae breakpoints $(\mathrm{S}=2, \mathrm{R}=8 \mu \mathrm{g} / \mathrm{mL})$; N/A indicates breakpoint was not available.

Table 5. In vitro activity of tigecycline and other antibiotics against resistant Gram-positive and Gram-negative organisms collected in Latin America from 2004-2007.

\begin{tabular}{|c|c|c|c|c|c|c|}
\hline Antibacterial & $\mathbf{N}$ & $\mathbf{M I C}_{50}$ & MIC $_{90}$ & $\% \mathrm{~S}^{*}$ & $\% \mathbf{R}$ & MICrange \\
\hline \multicolumn{7}{|l|}{ Gram-positives } \\
\hline \multicolumn{7}{|l|}{ MRSA } \\
\hline Tigecycline & 437 & 0.12 & 0.25 & 100 & N/A & $0.03-0.5$ \\
\hline Levofloxacin & 437 & 8 & 32 & 12.8 & 83.1 & $\leq 0.06-\geq 64$ \\
\hline Minocycline & 437 & $\leq 0.25$ & 4 & 98.4 & 0.2 & $\leq 0.25-\geq 16$ \\
\hline Linezolid & 437 & 2 & 2 & 100 & N/A & $\leq 0.5-4$ \\
\hline Vancomycin & 437 & 1 & 1 & 100 & 0.0 & $0.25-2$ \\
\hline \multicolumn{7}{|l|}{ VR E. faecium } \\
\hline Tigecycline & 47 & 0.06 & 0.12 & N/A & N/A & $0.03-0.25$ \\
\hline Penicillin & 47 & $\geq 16$ & $\geq 16$ & 4.3 & 95.7 & $4-\geq 16$ \\
\hline Ampicillin & 47 & $\geq 32$ & $\geq 32$ & 4.3 & 95.7 & $2-\geq 32$ \\
\hline Piperacillin-tazobactam & 47 & $\geq 32$ & $\geq 32$ & N/A & N/A & $16-\geq 32$ \\
\hline Imipenem $^{\S}$ & 22 & $\geq 32$ & $\geq 32$ & N/A & N/A & $8-\geq 32$ \\
\hline Levofloxacin & 47 & $\geq 64$ & $\geq 64$ & 0.0 & 100 & $16-\geq 64$ \\
\hline Minocycline & 47 & $\leq 0.25$ & $\geq 16$ & 74.5 & 17.0 & $\leq 0.25-\geq 16$ \\
\hline Linezolid & 47 & 2 & 2 & 97.9 & 0.0 & $1-4$ \\
\hline Vancomycin & 47 & $\geq 64$ & $\geq 64$ & 0.0 & 100 & $32-\geq 64$ \\
\hline \multicolumn{7}{|l|}{ Gram-negatives } \\
\hline \multicolumn{7}{|l|}{ Imipenem-R Acinetobacter } \\
\hline Tigecycline & 104 & 0.5 & 2 & N/A & N/A & $0.12-8$ \\
\hline Tigecycline & 104 & 0.5 & 2 & 97.1 & 1.0 & $0.12-8$ \\
\hline \multicolumn{7}{|c|}{ (Enterobacteriaceae breakpoint ${ }^{¥}$ ) } \\
\hline Ampicillin & 104 & $\geq 64$ & $\geq 64$ & N/A & N/A & $32-\geq 64$ \\
\hline Amoxicillin-clavulanic acid & 104 & $\geq 64$ & $\geq 64$ & N/A & N/A & $16-\geq 64$ \\
\hline Piperacillin-tazobactam & 104 & $\geq 256$ & $\geq 256$ & 1.9 & 95.2 & $4-\geq 256$ \\
\hline Ceftazidime & 104 & $\geq 64$ & $\geq 64$ & 6.7 & 89.4 & $\leq 8-\geq 64$ \\
\hline Ceftriaxone & 104 & $\geq 128$ & $\geq 128$ & 0.0 & 92.3 & $16-\geq 128$ \\
\hline Cefepime & 104 & $\geq 64$ & $\geq 64$ & 3.8 & 83.7 & $4-\geq 64$ \\
\hline Imipenem $^{\S}$ & 104 & $\geq 32$ & $\geq 32$ & 0.0 & 100 & $16-\geq 32$ \\
\hline Levofloxacin & 104 & 8 & $\geq 16$ & 4.8 & 68.3 & $0.25-\geq 16$ \\
\hline Amikacin & 104 & 64 & $\geq 128$ & 13.5 & 74.0 & $2-\geq 128$ \\
\hline Minocycline & 104 & $\leq 0.5$ & 2 & 98.1 & 1.0 & $\leq 0.5-16$ \\
\hline
\end{tabular}




\begin{tabular}{|c|c|c|c|c|c|c|}
\hline \multicolumn{7}{|l|}{$\overline{\text { ESBL+ K. pneumoniae }}$} \\
\hline Tigecycline & 280 & 0.5 & 2 & 93.6 & 1.8 & $0.12-8$ \\
\hline Ampicillin & 280 & $\geq 64$ & $\geq 64$ & 0.0 & 98.9 & $16-\geq 64$ \\
\hline Amoxicillin-clavulanic acid & 280 & 32 & $\geq 64$ & 12.9 & 61.8 & $2-\geq 64$ \\
\hline Piperacillin-tazobactam & 280 & 64 & $\geq 256$ & 35.0 & 42.9 & $0.25-\geq 256$ \\
\hline Imipenem $^{\S}$ & 185 & 0.5 & 1 & 99.5 & 0.5 & $\leq 0.06-16$ \\
\hline Levofloxacin & 280 & 8 & $\geq 16$ & 33.6 & 58.9 & $\leq 0.008-\geq 16$ \\
\hline Amikacin & 280 & 8 & $\geq 128$ & 76.8 & 14.3 & $\leq 0.5-\geq 128$ \\
\hline Minocycline & 280 & 4 & $\geq 32$ & 65.7 & 21.4 & $\leq 0.5-\geq 32$ \\
\hline \multicolumn{7}{|l|}{ ESBL+E. coli } \\
\hline Tigecycline & 194 & 0.25 & 0.5 & 100 & 0.0 & $0.06-2$ \\
\hline Ampicillin & 194 & $\geq 64$ & $\geq 64$ & 0.0 & 99.5 & $16-\geq 64$ \\
\hline Amoxicillin-clavulanic acid & 194 & 16 & 32 & 22.7 & 30.9 & $2-\geq 64$ \\
\hline Piperacillin-tazobactam & 194 & 8 & 32 & 86.1 & 3.6 & $0.25-\geq 256$ \\
\hline Imipenem $^{\S}$ & 110 & 0.25 & 0.5 & 99.1 & 0.0 & $\leq 0.06-8$ \\
\hline Levofloxacin & 194 & $\geq 16$ & $\geq 16$ & 9.8 & 84.5 & $0.015-\geq 16$ \\
\hline Amikacin & 194 & 4 & 16 & 94.3 & 3.1 & $\leq 0.5-\geq 128$ \\
\hline Minocycline & 194 & 4 & $\geq 32$ & 61.3 & 25.8 & $\leq 0.5-\geq 32$ \\
\hline
\end{tabular}

*Susceptibility was only determined when $\geq 10$ isolates were available; §Imipenem numbers are lower due to some isolates being tested with meropenem; 'VR E. faecalis are not reported here as only four were reported during the four study years (2 in 2005, 2 in 2006); "using Enterobacteriaceae breakpoints $(\mathrm{S}=2, \mathrm{R}=8 \mu \mathrm{g} / \mathrm{mL})$; N/A indicates breakpoint is not available.

high $(100 \%$ for tigecycline, vancomycin and linezolid and 98.4\% for minocycline) (Table 5).

Tigecycline was the most active agent against vancomycinresistant (VR) E. faecium, with a $\mathrm{MIC}_{90}$ of $0.12 \mu \mathrm{g} / \mathrm{mL}$ over the four study years (Table 5). Linezolid was also active against VR E. faecium, with a $\mathrm{MIC}_{90}$ of $2 \mu \mathrm{g} / \mathrm{mL}$ and $97.9 \%$ of isolates susceptible (Table 5).

Imipenem-resistant Acinetobacter spp. had reduced susceptibility to most agents, with the exceptions of tigecycline ( $\mathrm{MIC}_{90} 2 \mu \mathrm{g} / \mathrm{mL}$ ) and minocycline ( $\mathrm{MIC}_{90} 2 \mu \mathrm{g} / \mathrm{mL}$ ) (Table 5).

Tigecycline and imipenem were the most active agents against ESBL-positive K. pneumoniae, with 93.6 and 99.5\% of isolates susceptible, respectively $\left(\mathrm{MIC}_{90} \mathrm{~s}\right.$ of 2 and $1 \mu \mathrm{g} /$ $\mathrm{mL}$, respectively). Similarly, tigecycline and imipenem were the most active agents against ESBL-positive $E$. coli, with susceptibilities of 100 and $99.1 \%$, respectively, and $\mathrm{MIC}_{90} \mathrm{~s}$ of $0.5 \mu \mathrm{g} / \mathrm{mL}$ for each (Table 5 ).

\section{Discussion}

Several global surveillance studies are currently underway, including the SENTRY and SMART (Study for Monitoring Antimicrobial Resistance Trends) studies. These and other surveillance studies allow for the monitoring of global trends in resistance, as well as providing data on regional resistance and demographic trends. The T.E.S.T. study has been ongoing since 2004, collating global and local data on resistance trends among Gram-positive and Gram-negative organisms from nosocomial patients and community/outpatients. To date, 384 centers in 48 countries have contributed isolates to the T.E.S.T. global study.

Among the 33 centers contributing isolates in our study, 19 were located in Argentina and Mexico (11 and 8, respectively). Despite the fact that Brazil has the largest population of any country in Latin America, only two centers from Brazil provided isolates to this study. Thus, results for Latin America as a whole (44.3\% vancomycin resistance among E. faecium) are biased towards results from those countries contributing the most isolates, in this case Argentina and Mexico. The WHONET Program [10,11] has previously shown high rates of vancomycin resistance among $E$. faecium isolates in Argentina (25\%-33\%) and Chile (41\%-58.6\%) from 2003 to 2004. The regional collection bias in this study therefore does not appear to exaggerate the prevalence of vancomycinresistant $E$. faecium in Latin America.

The MRSA accounted for $48.3 \%$ of $S$. aureus isolates collected across Latin America during our study. This is a sizeable increase compared to previous studies, which report MRSA prevalences ranging from $26.5 \%$ to $38.6 \%$ (during the 1999-2000 winter season [9], from 1997-2001 [12], from 20002001 [13] or among clinical isolates in 2003 [14]). This disparity may be due in part to differences in countries contributing isolates to these studies: in Gales et al. [13], 11 centers in six countries contributed isolates (Argentina, Brazil, Chile, Colombia, Mexico and Venezuela); in Sader et al. [14], 10 centers in five undefined countries took part; and Mendes et al. [9] utilized isolates from 13 centers of three countries (Argentina, Brazil and Mexico). The study of Mendes et al. [9] focused on community-acquired respiratory tract organisms, which may account in part for the difference in susceptibility rates noted between that study and our current report.

MRSA rates varied widely between countries in this study, ranging from $16.0 \%$ in Jamaica to $77.3 \%$ in Puerto Rico. Similar results have previously been reported by Sader et al. [3], who found oxacillin resistance rates to vary significantly, even 
between hospitals in the same country. Although MRSA are typically cross-resistant to several classes of antibacterial agents, all $S$. aureus isolates identified in our study were susceptible to tigecycline.

The results in our study suggest that susceptibility among isolates of $E$. coli has decreased in Latin American in recent years. Sader et al. [12] examined susceptibility rates among $457 \mathrm{E}$. coli isolates from nosocomial and community-acquired infections in Latin America from 1997-2001. Of the eight antibacterial agents used in both studies, susceptibility rates were lower for six in our study; susceptibility was reduced by as much as $39.8 \%$ in the case of ampicillin. Imipenem susceptibility was identical in these two studies, while levofloxacin susceptibility was 3.2\% higher in our study.

Previous surveillance studies have shown reduced susceptibility rates in Latin America to commonly-used antibacterial agents. Gales et al. [15] reported that isolates of $P$. aeruginosa and Acinetobacter spp. from Latin America have reduced susceptibility rates to most antibacterial agents compared to isolates from other regions. These conclusions are supported by our study. Stelling et al. [16] examined susceptibility trends among $E$. coli isolates from 10 countries and found that isolation from Latin America was associated with increased nonsusceptibility for all six antimicrobial agents (cefepime, ceftazidime, ciprofloxacin, gentamicin, piperacillintazobactam and tobramycin).

As a result of the high frequencies of ESBLs reported in the SMART study from 2003-2004, Rossi et al. [17] have questioned the use of extended-spectrum cephalosporins as empirical therapy for intra-abdominal infections in some geographic areas. ESBL-positive E. coli have increased from $12.0 \%$ in the Rossi et al. study [17] to $20.8 \%$ in our study, while ESBL-positive K. pneumoniae increased from $27.6 \%$ to $36.7 \%$. These increases in ESBL rates over such a short period reiterate Rossi's concerns about the use of inappropriate empirical therapy in the treatment of infections caused by resistant organisms, especially given that appropriate empirical therapy has been directly linked to clinical success (i.e., 18).

Tigecycline has been shown here to be highly active against most organisms collected from Latin America, such as S. aureus (including MRSA), E. faecalis, E. faecium (including VR isolates), Acinetobacter spp. (including MDR isolates), Enterobacter spp., K. pneumoniae (including ESBL-positive isolates), E. coli (including ESBL-positive isolates) and $S$. marcescens. Isolates of $S$. aureus (including oxacillin-resistant isolates) and Enterococcus spp. collected in Latin America from 2000 to 2002 have previously been shown to be highly susceptible to tigecycline, with $\mathrm{MICs}_{90}$ of $0.5 \mu \mathrm{g} / \mathrm{mL}$ and $100 \%$ susceptibilities reported for both types of bacteria [19].

Tigecycline has previously been shown to be highly active against these same organisms on a global scale. Hoban et al. [20], in an early T.E.S.T. study report, presented data on 6,792 clinical isolates collected from 40 centers across Europe, North America and Asia in 2004. In this study, tigecycline was shown to be highly active against both
Gram-positive isolates (including E. faecalis, E. faecium and S. aureus) and Gram-negative isolates (including Acinetobacter baumannii, Enterobacter aerogenes, Enterobacter cloacae, E. coli and K. pneumoniae), with susceptibilities ranging from $91.3 \%$ among ESBL-positive K. pneumoniae isolates to $100 \%$ among E. coli (ESBLpositive and -negative), VR E. faecium and VR E. faecalis isolates. As in our study, tigecycline had little activity against $P$. aeruginosa.

Bacterial resistance to many commonly-used antibacterial agents is increasing. This is particularly true in Latin America, where local antibacterial usage patterns and/or dissemination of resistant clones have led to increased resistance rates [4]. The resistance scenario in Latin America is further complicated by the recent appearance of metallo- $\beta$-lactamases among isolates of $A$. baumannii, $P$. aeruginosa and $K$. pneumoniae [21]. The development of new antibacterial agents that work through novel mechanisms, and are thus not affected by existing mechanisms of resistance, is critically needed. Tigecycline is one such agent that has been shown to be effective against most of the organisms identified in our study, even those resistant to other agents. Tigecycline may thus become an important tool in the treatment of infections caused by Gram-positive and Gram-negative organisms, including resistant strains.

\section{Acknowledgements}

The authors thank the many investigators who contributed isolates to this study. We also thank the staff of International Health Management Associated, Inc., Schaumburg, IL, USA for coordination of the T.E.S.T study.

This study was financially supported by Wyeth Pharmaceuticals. Medical writing support was provided by Micron Research Ltd; this collaboration was also supported by Wyeth Pharmaceuticals.

\section{References}

1. Deshpande L.M., Fritsche T.R., Moet G.J., et al. Antimicrobial resistance and molecular epidemiology of vancomycin-resistant enterococci from North America and Europe: a report from the SENTRY antimicrobial surveillance program. Diagn Microbiol Infect Dis 2007;58(2):163-70.

2. Falagas M.E., Karveli E.A. The changing global epidemiology of Acinetobacter baumannii infections: a development with major public health implications. Clin Microbiol Infect 2007;13:179.

3. Sader H.S., Jones R.N., Gales A.C., et al. SENTRY antimicrobial surveillance program report: Latin America and Brazilian results for 1997 through 2001. Braz J Infect Dis 2004;8:25-79.

4. Andrade S.S., Sader H.S., Jones R.N., et al. Increased resistance to first-line agents among bacterial pathogens isolated from urinary tract infections in Latin America: time for local guidelines? Memórias de Instituto Oswaldo Cruz, Rio de Janeiro 2006:101;741-8.

5. Felmingham D. The need for antimicrobial resistance surveillance. J Antimicrob Chemother 2002;50(suppl. S1):1-7.

6. National Committee for Clinical Laboratory Standards. Methods for dilution antimicrobial susceptibility tests for bacteria that grow aerobically, document M7-A6. NCCLS, 2003. 
7. Wyeth Pharmaceuticals Inc. Tygacil Product Insert. Philadelphia, PA, USA, 2007. http://www.tygacil.com (last accessed 24 April 2007).

8. Clinical and Laboratory Standards Institute. Performance standards for antimicrobial susceptibility testing, $16^{\text {th }}$ informational supplement, document M100-S16. CLSI, 2006.

9. Mendes C., Marin M.E., Quiñones F., et al. Antibacterial resistance of community-acquired respiratory tract pathogens recovered from patients in Latin America : results from the PROTEKT Surveillance Study (1999-2000). Braz J Infect Dis 2003;7:44-61.

10. Informe Anual de la Red de Monitoreo/Vigilancia de la Resistencia a los Antibióticos, 2003. Organización Panamericana de la Salud, Oficina Regional de la Organización Mundial de la Salud (OPS/ DPC/CD/332/05).

11. Informe Anual de la Red de Monitoreo/Vigilancia de la Resistencia a los Antibióticos, 2004 (Brasilia, Brasil 27 al 29 de julio, 2005). Organización Panamericana de la Salud, Oficina Regional de la Organización Mundial de la Salud (OPS/HDM/CD/A/408/06).

12. Sader H.S., Jones R.N., Gales A.C., et al. SENTRY Antimicrobial Surveillance Program Report: Latin American and Brazilian Results for 1997 through 2001. Braz J Infect Dis 2004;8:25-79.

13. Gales A.C., Andrade S.S., Sader H.S., Jones R.N. Activity of mupirocin and 14 additional antibiotics against staphylococci isolated from Latin American hospitals: report from the SENTRY antimicrobial surveillance program. J Chemother 2004;16:323-8.

14. Sader H.S., Fritsche T.R., Streit J.M., Jones R.N. Daptomycin in vitro activity tested against Gram-positive strains collected from European and Latin American centers in 2003. J Chemother 2005; $17: 477-83$.
15. Gales A.C., Jones R.N. and Sader H.S. Global assessment of the antimicrobial activity of polymixin B against 54731 isolates of Gram-negative bacilli: report from the SENTRY antimicrobial surveillance program 2001-2004. Clin Microbiol Infect 2006;12:315-21.

16. Stelling J.M., Travers K., Jones R.N., et al. Integrating Escherichia coli antimicrobial susceptibility data from multiple surveillance programs. Emerg Infect Dis 2005;11:873-82.

17. Rossi F., Baquero F., Hsueh P.-R., et al. In vitro susceptibilities of aerobic and facultative anaerobic Gram-negative bacilli isolated from patients with intra-abdominal infections worldwide: 2004 results from SMART (Study for Monitoring Antimicrobial Resistance Trends). J Antimicrob Chemother 2006;58:205-10.

18. Krobot K., Yin D., Zhang Q., et al. Effect of inappropriate initial empiric antibiotic therapy on outcome of patients with community-acquired intra-abdominal infections requiring surgery. Eur J Clin Microbiol Infect Dis 2004;23:682-7.

19. Gales A.C., Jones R.N., Andrade S.S., Pereira A.S., Sader H.S. In vitro activity of tigecycline, a new glycylcycline, tested against 1,326 clinical bacterial strains isolated from Latin America. Braz J Infect Dis 2005:9;348-56.

20. Hoban D.J., Bouchillon S.K., Johnson B.M., et al. In vitro activity of tigecycline against 6792 Gram-negative and Gram-positive clinical isolates from the global Tigecycline Evaluation and Surveillance Trial (TEST Program, 2004). Diagn Microbiol Infect Dis 2005:52;215-27.

21. Lincopan N., McCulloch JA, Reinert C., et al. First isolation of metallo- $\beta$-lactamase-producing multiresistant Klebsiella pneumoniae from a patient in Brazil. J Clin Microbiol 2005;43:516-9. 